\title{
Electron beams generated by shock waves in the solar corona
}

\author{
G. Mann ${ }^{1}$ and A. Klassen ${ }^{2}$ \\ 1 Astrophysikalisches Institut Potsdam, An der Sternwarte 16, 14482 Potsdam, Germany \\ e-mail: GMann@aip.de \\ ${ }^{2}$ Institut für Experimentelle und Angewandte Physik, Universtät Kiel, 21118 Kiel, Germany
}

Received 25 September 2003 / Accepted 13 April 2005

\begin{abstract}
Beams of energetic electrons can be generated by shock waves in the solar corona. At the Sun shock waves are produced either by flares and/or by coronal mass ejections (CMEs). They can be observed as type II bursts in the solar radio radiation. Shock accelerated electron beams appear as rapidly drifting emission stripes (so-called "herringbones") in dynamic radio spectra of type II bursts. A large sample of type II bursts showing "herringbones" was statistically analysed with respect to their properties in dynamic radio spectra. The electron beams associated with the "herringbones" are considered to be generated by shock drift acceleration. Then, the accelerated electrons establish a shifted loss-cone distribution in the upstream region of the associated shock wave. Such a distribution causes plasma instabilities leading to the emission of radio waves observed as "herringbones". Consequences of a shifted loss-cone distribution of the shock accelerated electrons are discussed in comparison with the observations of "herringbones" within solar type II radio bursts.
\end{abstract}

Key words. acceleration of particles - magnetic fields - Sun: radio radiation

\section{Introduction}

The Sun is a giant source of energetic electrons (Lin 1974; Kahler 1994; Reames et al. 1996; Klein \& Trottet 2001). That play a particular role, since they are the source of non-thermal radio and X-ray radiation. Electrons can be accelerated by the electric field induced by magnetic reconnection (Holman 1985; Benz 1987; Litvinenko 2000), shock waves (Holman \& Pesses 1983; Schlickeiser 1984; Kirk 1994; Mann \& Classen 1995; Mann et al. 2001), and stochastic acceleration via wave particle interaction (Melrose 1994; Miller 1997). Shock accelerated electron beams, in particular, are the subject of the presented paper.

In the solar corona, shock waves can occur as blast waves due to the flare process (Uchida et al. 1973; Vršnak et al. 1995) or as shocks driven by coronal mass ejections (CMEs) (Stewart et al. 1974a,b). These shocks can be observed as type II bursts in the solar radio radiation in the meteric wave range (Wild \& McCready 1950; Uchida 1960). They appear as stripes of enhanced radio emission slowly drifting from high to low frequencies in dynamic radio spectra (see Nelson \& Melrose 1985; Mann 1995; Aurass 1996; and Cairns et al. 2004, for reviews). A particular example is presented in Fig. 1. Generally, the type II bursts show two different components. The so-called "backbone" is slowly drifting from high to low frequencies with typical drift rates of $\approx-0.1 \mathrm{MHz} / \mathrm{s}$ (Mann 1995), and is considered as the signature of the associated shock wave travelling outwards through the corona (Nelson $\&$ Melrose 1985). The second component appears as rapidly drifting $(\approx \pm 10 \mathrm{MHz} / \mathrm{s})$ stripes of enhanced radio emission shooting up from the "backbone" towards both high and low frequencies. These features are called "herringbones" (Roberts 1959) and recall type III radio bursts (Suzuki \& Dulk 1985). Therefore, the "herringbones" are regarded as signatures of electron beams, which are accelerated by the shock wave associated with the "backbone" (Nelson \& Melrose 1985). However there are some morphological differences between type III bursts and "herringbones" (Cairns \& Robinson 1987; Zlobec et al. 1993). Recently, Mann \& Klassen (2002) investigated a large sample of type III bursts and "herringbones" with respect to their drift rate in the same frequency range and found a mean ratio of 1.92 between the amount of the drift rate of type III bursts and those of "herringbones"; i.e. the drift rates of "herringbones" are approximately a half of those of type III bursts in a statistical sense.

Recently, Bale et al. (1999) reported that the source of an interplanetary type II radio burst is located at regions, where the shock is nearly perpendicular; i.e. the angle between the upstream magnetic field and the shock normal is nearly $90^{\circ}$. For such shocks, shock drift acceleration is very efficient for accelerating electrons up to supra-thermal energies (Holman $\&$ Pesses 1983). Such electrons are even needed to generate the non-thermal radio radiation of type II bursts. On the basis of observations in Bale et al. (1999), Knock et al. (2001, 2003) presented a complete model of type II radio bursts associated with solar and/or interplanetary shocks. Here, they considered that the energetic electrons are produced by shock drift acceleration establishing a shifted loss-cone distribution 


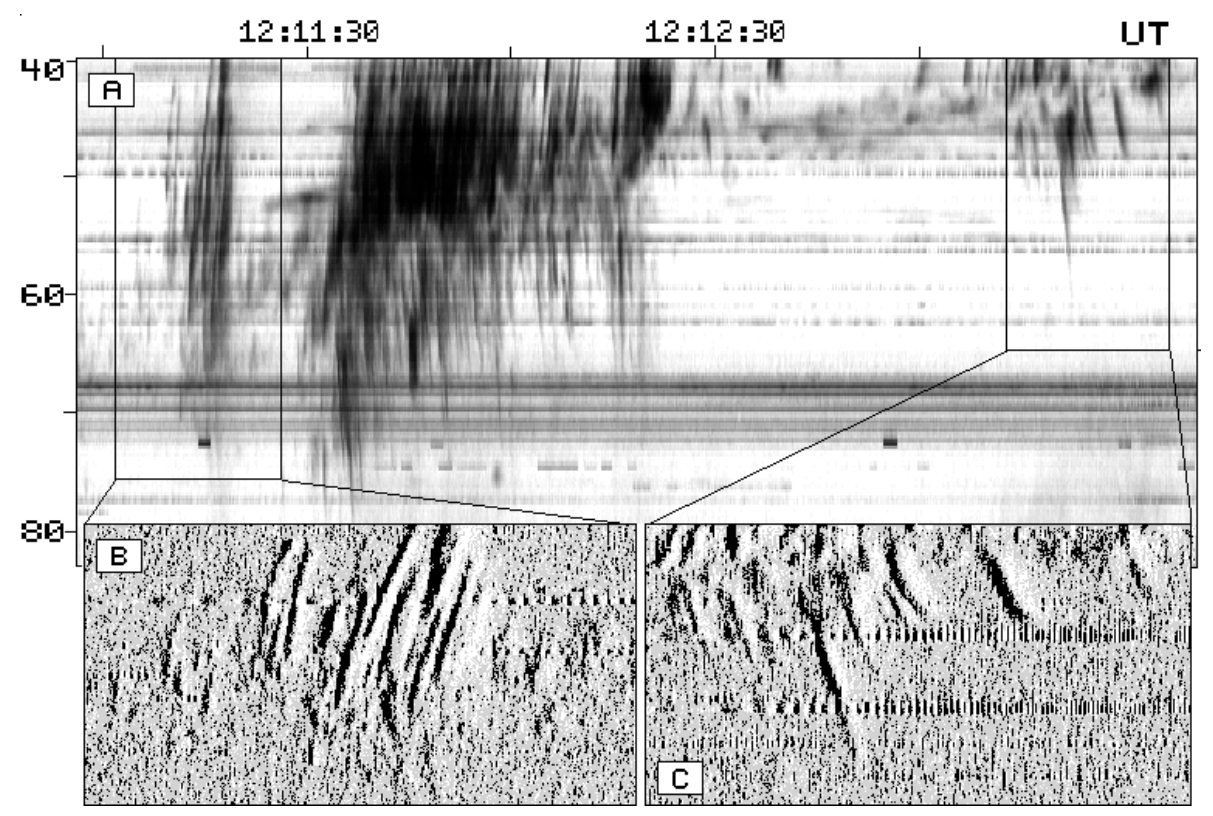

Fig. 1. Dynamic radio spectrum (A) of the solar type II burst during the event on June 30, 1995. The "backbone" is slowly drifting from 60 to $42 \mathrm{MHz}$. The "herringbones" are nicely visible during the whole event. The radio intensity is coded by grey scale. The bottom frames (B, C) present the intensity time derivative of the individual "herringbones".

(Leroy \& Mangeney 1984; Wu 1984) of the electrons in the upstream region. Such a distribution is unstable for exciting high frequency plasma (e.g. Langmuir) waves, which convert into escaping radio waves at the fundamental and harmonic emission frequency by stochastic growth theory (Cairns \& Robinson 1997, 1999, 2000). Finally, they calculated synthetic radio spectra, which could be observed by any observer at any location in the heliosphere.

The aim of the paper is to discover what can be learnt from observations for a better understanding of shock accelerated electron beams in the solar corona, if one adopts shock drift acceleration as the basic process. In doing that, a sample of 11 solar type II radio bursts with 144 "herringbones" are studied with respect to their properties (e.g. drift rates) in the dynamic radio spectra in Sect. 2. In Sect. 3 the results of these observations are briefly discussed. Here, the electron beams associated with the "herringbones" are regarded as produced by shock drift acceleration leading to a so-called shifted loss-cone distribution of these electrons (Sect. 4). The consequences of such a distribution are compared to the observations of "herringbones" in dynamic radio spectra in Sect. 5, and results are summarized in Sect. 6.

\section{Observations}

In order to investigate "herringbones", 11 solar type II radio bursts were selected in the period 1994-1997 (see Table 1), since they show "herringbones" that occur at the well-observed frequency $f_{\text {st }}$ and disappear at $f_{\text {end }}$. Thus, a sample of 144 individual "herringbones" with both a positive (74 cases) and negative ( 70 cases) drift is available.

All these radio bursts were recorded by the radiospectralpolarimeter (Mann et al. 1992) of the Astrophysical Institute Potsdam (Germany). It covers a frequency range $40-800 \mathrm{MHz}$
Table 1. Summary of the solar type II radio burst events.

\begin{tabular}{lccc}
\hline \hline Date of event & Range (MHz) & Band & $\left|D_{f, \mathrm{II}}\right|(\mathrm{MHz} / \mathrm{s})$ \\
\hline June 24, 1994 & $78-57$ & $F$ & $0.17-0.19$ \\
July 7, 1994 & $81-51$ & $F$ & $0.16-0.19$ \\
July 18, 1994 & $80-51$ & $H$ & 0.095 \\
Febr. 21, 1995 & $76-43$ & $H$ & $0.067-0.075$ \\
March 6, 1995 & $65-48$ & $H$ & $0.063-0.085$ \\
June 30, 1995 & $66-43$ & $F$ & $0.171-0.145$ \\
Aug. 22, 1996 & $75-42$ & $H$ & 0.125 \\
Dec. 24, 1996 & $59-42$ & $F$ & $0.160-0.170$ \\
April 2, 1997 & $80-50$ & $H$ & $0.11-0.12$ \\
May 12, 1997 & $73-50$ & $H$ & $0.12-0.18$ \\
Sept. 17, 1997 & $67-52$ & $F$ & $0.120-0.136$ \\
\hline
\end{tabular}

with a temporal resolution of $0.1 \mathrm{~s}$. The spectral resolution $\Delta f / f$ is a few $100 \mathrm{kHz}$. Thus, both resolutions are better than those of the Culgoora spectrograph (i.e. temporal resolution $0.5 \mathrm{~s}$ and spectral resolution $1 \mathrm{MHz}$ ) whose data was used in the study by Cairns \& Robinson (1997).

In Table 1 ( 3 rd column), $\mathrm{F}$ and $\mathrm{H}$ denote the fundamental and harmonic emissions, respectively. The drift rates of the "backbone" $D_{f, \text { II }}$ and the "herringbones" $D_{f, \mathrm{HB}}$ are generally normalized with respect to the fundamental emission. This sample yields the following average parameters:

- start frequency of a "herringbone" $f_{\mathrm{st}}=54.0 \mathrm{MHz}$;

- whole frequency range of a "herringbone" $\Delta f_{\mathrm{HB}}=f_{\text {end }}-$ $f_{\mathrm{st}}=10.1 \mathrm{MHz}$ (i.e. $\Delta f_{\mathrm{HB}} / f_{\mathrm{st}}=0.19$ );

- whole duration of a "herringbone" $\Delta t=t_{\mathrm{end}}-t_{\mathrm{st}}=1.36 \mathrm{~s}$;

- drift rate of a "backbone" $\left|D_{f, \text { II }}\right|=0.133 \mathrm{MHz} / \mathrm{s}$ (i.e. $\left.\left|D_{f, \mathrm{II}}\right| / f_{\text {st }}=0.0025 \mathrm{~s}^{-1}\right)$;

- drift rate of a "herringbone" $\left|D_{f, \mathrm{HB}}\right|=9.1 \mathrm{MHz} / \mathrm{s}$ (i.e. $\left.\left|D_{f, \mathrm{HB}}\right| / f_{\mathrm{st}}=0.17 \mathrm{~s}^{-1}\right)$. 


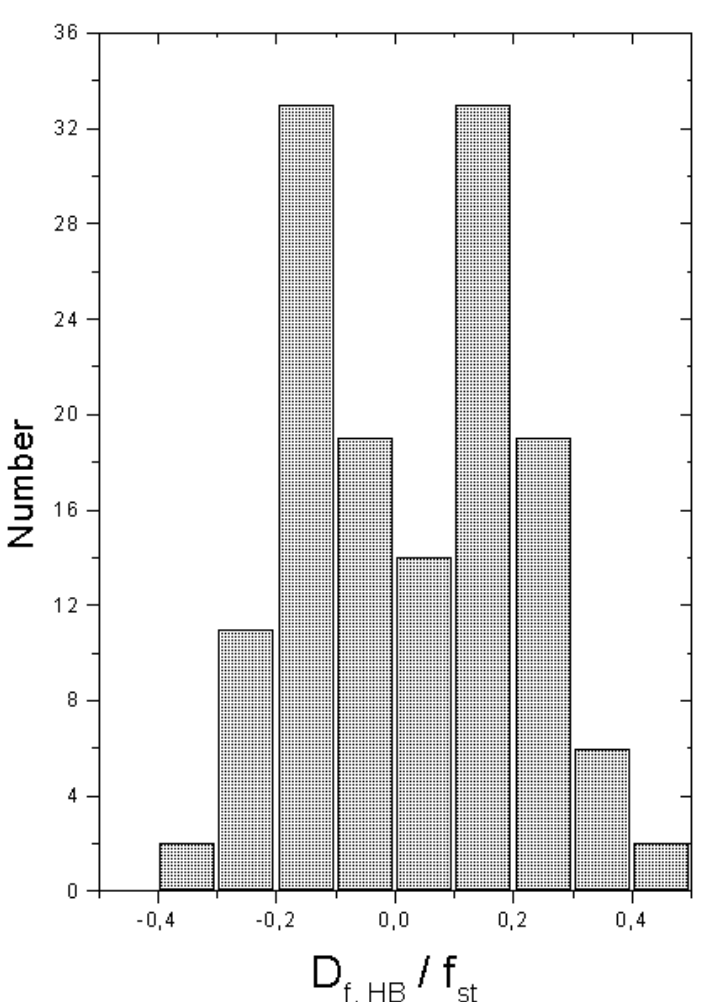

Fig. 2. Distribution of the ratios $D_{f, \mathrm{HB}} / f_{\mathrm{st}}$ given in $\mathrm{s}^{-1}$ for the whole sample of "herringbones".

Figure 2 offers the distribution of the amount of ratio $D_{f, \mathrm{HB}} / f_{\mathrm{st}}$ between the drift rates $D_{f, \mathrm{HB}}$ and the corresponding start frequency $f_{\text {st }}$ for the whole sample of "herringbones". They cover a range $0.03-0.49 \mathrm{~s}^{-1}$ with a mean value of $0.17 \mathrm{~s}^{-1}$. Additionally, Fig. 3 presents the distribution of the ratio of the amount of the drift rate of the individual "herringbones" to that of the associated "backbone". It shows a sharp maximum at $50-75$. This ratio has a mean value of 77 . The scatter plot of the duration $\Delta t$ of an individual "herringbone" versus $f_{\mathrm{st}} /\left|D_{f, \mathrm{HB}}\right|$ is presented in Fig. 4. The duration $\Delta t$ of a "herringbone" is determined as the time difference, where it starts at $f_{\text {st }}$ and, subsequently, disappears at $f_{\text {end }}$ in the dynamic radio spectrum. The linear regression analysis provides

$\Delta t=A+B \cdot \frac{f_{\mathrm{st}}}{\left|D_{f, \mathrm{HB}}\right|}$

with $A=0.34 \mathrm{~s}$ and $B=0.14$, as well as a correlation coefficient of 0.84 .

\section{Interpretation}

In the solar corona the nonthermal radio radiation in the meter wave range is generally assumed to be generated by plasma emission, meaning that energetic electrons excite high frequency plasma waves (e.g. Langmuir and/or upper hybrid waves), which nonlinearly convert into escaping radio waves. Consequently, the radio emission takes place near the local electron plasma frequency $f_{\mathrm{pe}}=\left(e^{2} N / \pi m_{\mathrm{e}}\right)^{1 / 2}(e$, elementary charge; $N$, electron number density; $m_{\mathrm{e}}$, electron mass) and/or

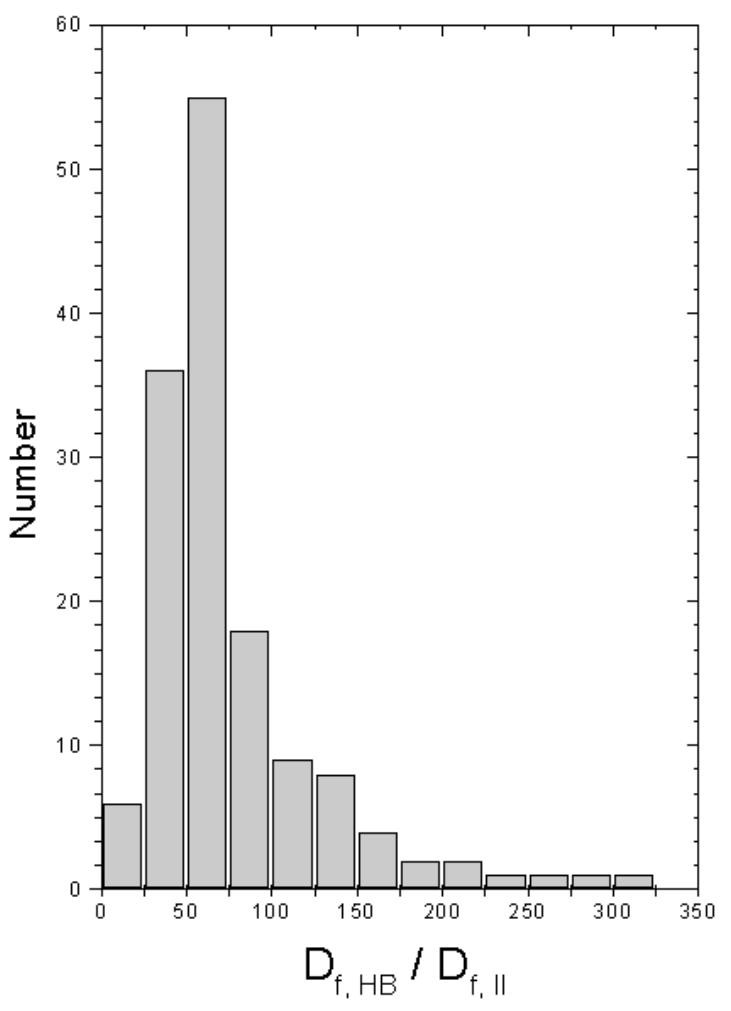

Fig. 3. Distribution of the amount of the drift rate of the "herringbones" to that of the associated "backbone" for the whole sample.

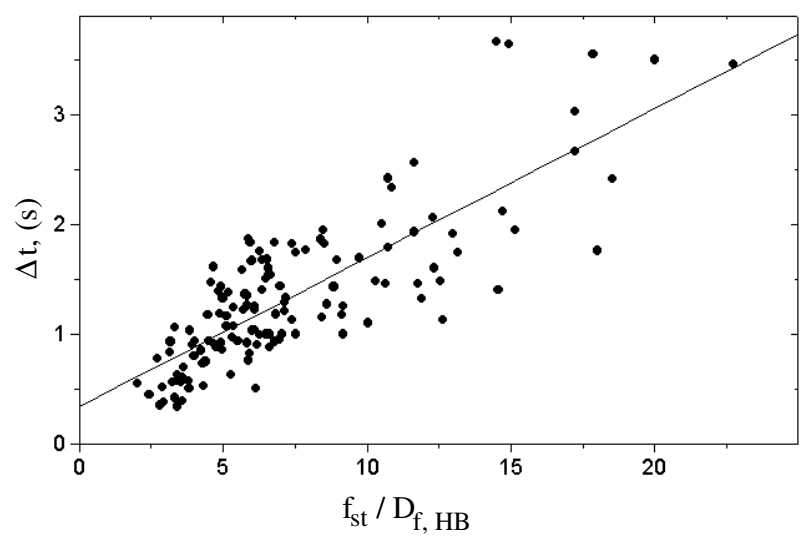

Fig. 4. Scatter plot of the duration of the "herringbones" versus $f_{\mathrm{st}} /\left|D_{f, \mathrm{HB}}\right|$. The solid line represents the linear regression.

its harmonics (Melrose 1985). If a radio source is moving upor downwards in the solar corona, it causes a frequency drift to lower or higher frequencies in the dynamic radio spectrum due to density inhomogeneity in the corona, respectively. Then, the drift rate $D_{f}$ at the observed frequency $f \approx v f_{\text {pe }}(v$, number of harmonics) is related to the velocity $V_{\text {source }}$ of the radio source by

$D_{f}=\frac{\mathrm{d} f}{\mathrm{~d} t}=\frac{f}{2} \cdot \frac{1}{N} \cdot \frac{\mathrm{d} N}{\mathrm{~d} R} \cdot V_{\text {source }} \cdot \cos \vartheta$

with the radial density gradient $\mathrm{d} N / \mathrm{d} R$. Here, $\vartheta$ denotes the angle between the propagation direction of the radio source 
and the radial one. The assumption of a hydrostatic, isothermal equilibrium of a gravitaionally stratified corona results in a density model (see e.g. Mann et al. 1999b)

$N(R)=N_{\mathrm{S}} \cdot \mathrm{e}^{D\left(R_{\mathrm{S}} / R-1\right)}$

with $D=\tilde{\mu} m_{\mathrm{p}} M_{\mathrm{S}} G / k_{\mathrm{B}} T R_{\mathrm{S}}\left(\tilde{\mu}\right.$, mean molecular weight; $m_{\mathrm{p}}$, proton mass; $M_{\mathrm{S}}$, mass of the Sun; $G$, gravitational constant; $k_{\mathrm{B}}$, Boltzmann's constant; $T$, temperature; $R_{\mathrm{S}}$, radius of the Sun). In the case of an electron number density of $N_{\mathrm{S}}=$ $8.775 \times 10^{8} \mathrm{~cm}^{-3}$ at the base of the corona and a coronal temperature of $T=1.4 \times 10^{6} \mathrm{~K}$, i.e. $D=9.88$ with $\tilde{\mu}=0.6$, the density model of Eq. (3) agrees with the one-fold Newkirk (1961) model, which is quite able to describe the density behaviour in the corona above quiet equatorial regions (Koutchmy 1994). Yohkoh and SOHO images of the Sun and complementary spatial radio-heliographic observations revealed that solar type II radio burst sources are established near but outside of active regions (Aurass et al. 1998; Klassen et al. 1999; Klein et al. 1999; Gopalswamy et al. 2000). Therefore, a one-fold Newkirk (1961) model is chosen as an appropriate density model for describing the coronal regions in the environs of type II burst sources (Mann et al. 2003). Then, the radial component of the radio source velocity $V_{r \text {,source }}$ can be determined from the drift rate by

$V_{r, \text { source }}=\frac{\left(-2 D_{f}\right)}{f} \cdot \frac{R_{\mathrm{S}}}{D} \cdot\left(\frac{R}{R_{\mathrm{S}}}\right)^{2}$

according to Eq. (3), where the radial distance $R$ of the electron number density $N$ is given by

$$
\frac{R}{R_{\mathrm{S}}}=\left[1+\frac{1}{A} \cdot \ln \left(\frac{N}{N_{\mathrm{S}}}\right)\right]^{-1} .
$$

Note, that the radial source velocity $V_{r \text {,source }}$ is proportional to the ratio $D_{f} / f$, which is why the "herringbones" were investigated for this quantity (see Fig. 2).

The mean start frequency of the "herringbones" is about $54 \mathrm{MHz}$, which corresponds to an electron number density of $3.62 \times 10^{7} \mathrm{~cm}^{-3}$. Such a density is found at a radial distance of $1.48 R_{\mathrm{S}}$ from the center of the Sun (see Eq. (5)), where a magnetic field of $1.5 \mathrm{G}$ is expected according to Dulk \& McLean (1978) resulting in a local Alfvén speed $v_{\mathrm{A}}=510 \mathrm{~km} \mathrm{~s}^{-1}$.

According to Eqs. (4) and (5) the mean radial velocity of the type II burst source is $V_{r, \text { II }}=760 \mathrm{~km} \mathrm{~s}^{-1}$, i.e. the Alfvén-Mach number $M_{\mathrm{A}}=v_{\mathrm{s}} / v_{\mathrm{A}}>1.5$ with $v_{\mathrm{s}}$ as the shock speed. The radial velocity of the "herringbone" sources is in the range $9200-150000 \mathrm{~km} \mathrm{~s}^{-1}$ with a mean value of $V_{r, \mathrm{HB}}=$ $52000 \mathrm{~km} \mathrm{~s}^{-1}=0.17 \mathrm{c}$ (c, velocity of light). This corresponds to kinetic energies $0.2-80 \mathrm{keV}$ with a mean value at $7 \mathrm{keV}$, which agrees with values recently found for other type II bursts (Klassen et al. 2000) and "herringbones" (Mann \& Klassen 2002 ) in the frequency range $40-100 \mathrm{MHz}$. In contradiction to Fig. 3 in the paper by Cairns \& Robinson (1987) no "herringbones" with radial source velocities above $0.5 \mathrm{c}$ was observed in the presented sample.

Figure 3 shows the distribution of ratio $D_{f, \mathrm{HB}} / D_{f, \mathrm{II}}$ for the whole sample. It has a relatively sharp maximum around 63 .
According to Eq. (4), this quantity ratio gives the ratio between the velocity of the electrons related to the "herringbones" and that of the shock wave associated to the type II burst. Therefore, a faster shock is generating electron beams with higher velocities.

Figure 4 reveals a relationship between the duration of a "herringbone" to the quantity $f_{\mathrm{st}} / D_{f, \mathrm{HB}}$ expressed by Eq. (1). Inserting $R=1.48 R_{\mathrm{S}}$ into Eq. (4) as the radial distance of the $54 \mathrm{MHz}$ level, Eq. (1) leads to $V_{r, \mathrm{HB}} \cdot \Delta t=\Delta r_{\mathrm{HB}}=$ $43400 \mathrm{~km}+\left(V_{r, \mathrm{HB}} \cdot A\right)$. Since radial velocities of the "herringbone" sources are in the range 9200-150000 $\mathrm{km} \mathrm{s}^{-1}$ (compare with Fig. 4), $\Delta r_{\mathrm{HB}}$ varies between 46000 and $94000 \mathrm{~km}$, making it a weakly increasing function of $V_{r, \mathrm{HB}}$. Such a relationship was already found by Cairns \& Robinson (1987). Since $\Delta r_{\mathrm{HB}}$ means the radial travel distance of the shock accelerated electron beam from the shock surface, this distance only weakly depends on the radial beam velocity. Note that Cairns \& Robinson (1987) found travel distances up to $1 R_{\mathrm{S}}$ (see Fig. 3 in their paper). Such large travel distances have not been observed in the sample basically used in the present study.

\section{Intoduction to shock drift acceleration}

Fast magnetosonic shock waves are accompanied by compression of the magnetic field. Thus, it represents a moving magnetic mirror, at which charged particles can be reflected and accelerated. Therefore, the electron beams associated with the "herringbones" are regarded as being generated by shock drift acceleration. Since the electrons related to the "herringbones" have typical energies of about $7 \mathrm{keV}$, they can be treated as non-relativistic particles.

In order to present a brief introduction to shock drift acceleration the reader is referred to the paper by Ball \& Melrose (2001) for a much more detailed description. The velocity gain due to shock-drift acceleration is given by

$V_{r, \|}=2 v_{\mathrm{s}} \sec \theta-V_{i, \|}$

(Holman \& Pesses 1983). Here, $V_{i, \|}$ and $V_{r, \|}$ denote the particle velocity parallel to the ambient magnetic field before and after the reflection process, respectively. $\theta$ is the angle between the upstream magnetic field $\boldsymbol{B}_{\text {up }}$ and the shock normal $\boldsymbol{n}_{\mathrm{s}}$. The particle velocity perpendicular to the magnetic field stays unchanged during the reflection process

$V_{r, \perp}=V_{i, \perp}$.

Usually, the reflection process is treated in the de HoffmannTeller frame, in which the shock wave is at rest and the motional electric field is removed (Ball \& Melrose 2001). There, the reflection takes place under the conservation of energy

$\frac{m_{\mathrm{e}}}{2} \cdot\left(V_{i, \|, \mathrm{HT}}^{2}+V_{i, \perp, \mathrm{HT}}^{2}\right)=\frac{m_{\mathrm{e}}}{2}\left(V_{\|, \mathrm{HT}}^{2}+V_{\perp, \mathrm{HT}}^{2}\right)-e \phi_{\mathrm{HT}}$

and the magnetic moment

$\frac{V_{i, \perp, \mathrm{HT}}^{2}}{B_{\text {up }}}=\frac{V_{\perp, \mathrm{HT}}^{2}}{B_{\max }}$.

Here, all components of the particle velocity, as well as the cross shock potential $\phi_{\mathrm{HT}}$, are given in the de Hoffmann-Teller 
frame. Note that the cross shock potential is generally frame dependent, and $B_{\max }$ is the maximum of the magnitude of the magnetic field at the shock transition.

In the framework of the two fluid plasma model (Krall \& Trivelpiece 1973), the cross shock potential can be determined by means of the momentum equation of the electron fluid neglecting the electron mass. Then, the cross shock potential is given by

$e \phi_{\mathrm{HT}}=\frac{\gamma}{(\gamma-1)} \cdot k_{\mathrm{B}} T \cdot\left[\left(\frac{N}{N_{0}}\right)^{(\gamma-1)}-1\right]$

( $k_{\mathrm{B}}$, Boltzmann's constant; $\gamma$, ratio of the specific heat) in the de Hoffmann-Teller frame (Goddrich \& Scudder 1984; Kunic et al. 1999). $N / N_{0}$ denotes the jump in density across the shock, where $T$ and $N_{0}$ are the temperature and particle number density in the far upstream region. Furthermore, $\phi_{\mathrm{HT}}=0$ and quasi-neutrality were assumed in the far upstream region. In the isothermal limit, i.e. $\gamma \rightarrow 1$, one gets $e \phi=k_{\mathrm{B}} T \cdot \ln \left(N / N_{0}\right)$ for the electrostatic cross shock potential.

Now, the conditions under which the reflection takes place are derived by using Eqs. (8) and (9) leading to

$V_{\|, \mathrm{HT}}^{2}=V_{i, \|, \mathrm{HT}}^{2}-\left(\frac{B_{\mathrm{max}}}{B_{\mathrm{up}}}-1\right) V_{i, \perp, \mathrm{HT}}^{2}+\frac{e \phi_{\mathrm{HT}}}{m_{\mathrm{e}}}$.

If the particle should be reflected, $V_{\|, \mathrm{HT}}$ must vanish anywhere at the shock. Consequently, all particles fulfilling the condition

$V_{i, \perp, \mathrm{HT}}>\tan \alpha_{\mathrm{lc}} \cdot \sqrt{V_{i, \|, \mathrm{HT}}^{2}+\frac{2 e \phi_{\mathrm{HT}}}{m_{\mathrm{e}}}}$

are reflected at the shock wave. The so-called loss-cone angle $\alpha_{\mathrm{lc}}$ is defined by $\alpha_{\mathrm{lc}}=\arcsin \left[\left(B_{\mathrm{up}} / B_{\max }\right)^{1 / 2}\right]$. The velocity components in the de Hoffmann-Teller frame are related to those in the laboratory frame by

$V_{\|, \mathrm{HT}}=V_{\|}-v_{\mathrm{s}} \sec \theta$

$V_{\perp, \mathrm{HT}}=V_{\perp}$

(Ball \& Melrose 2001). Finally, the particles establish a socalled shifted loss-cone distribution function

$$
\begin{aligned}
f_{\mathrm{acc}}= & \Theta\left(V_{\|}-v_{\mathrm{s}} \sec \theta\right) \\
& \times \Theta\left(V_{\perp}-\tan \alpha_{\mathrm{lc}} \cdot \sqrt{\left(V_{\|}-v_{\mathrm{s}} \sec \theta\right)^{2}+\frac{2 e \phi_{\mathrm{HT}}}{m_{\mathrm{e}}}}\right) \\
& \times f_{0}\left(2 v_{\mathrm{s}} \sec \theta-V_{\|}, V_{\perp}\right)
\end{aligned}
$$

after the reflection in the upstream region if $f_{0}\left(V_{\|}, V_{\perp}\right)$ is the initial one (Leroy \& Mangeney 1984; Wu 1984).

Assuming a Maxwellian distribution function as the intitial state in the upstream region with thermal electron speed $v_{\text {th }}=\left(k_{\mathrm{B}} T / m_{\mathrm{e}}\right)^{1 / 2}$, the integration of $f_{\text {acc }}\left(V_{\|}, V_{\perp}\right)$ over the velocity component $V_{\perp}$ leads to the so-called reduced distribution function

$$
\begin{aligned}
F_{\mathrm{acc}}= & \frac{\Theta\left(V_{\|}-V_{\mathrm{s}}\right)}{\left(2 \pi v_{\mathrm{th}}^{2}\right)^{1 / 2}} \cdot \mathrm{e}^{-\left[V_{\mathrm{s}}^{2} \sin ^{2} \alpha_{\mathrm{lc}}+V_{\mathrm{e}}^{2} \tan ^{2} \alpha_{\mathrm{lc}}\right] / 2 v_{\mathrm{th}}^{2}} \\
& \times \exp \left(-\frac{\left[V_{\|}-V_{\mathrm{s}}\left(1+\cos ^{2} \alpha_{\mathrm{lc}}\right)\right]^{2}}{2 v_{\mathrm{th}}^{2} \cos ^{2} \alpha_{\mathrm{lc}}}\right)
\end{aligned}
$$

with $V_{\mathrm{s}}=v_{\mathrm{s}} \sec \theta$ and $V_{\mathrm{e}}=\left(2 e \phi_{\mathrm{HT}} / m_{\mathrm{e}}\right)^{1 / 2}$. It only depends on the velocity component $V_{\|}$and actually represents a distribution function of an electron beam with the velocity $V_{\mathrm{b}}=V_{\mathrm{s}}(1+$ $\left.\cos ^{2} \alpha_{\mathrm{lc}}\right)$ and the width $2^{1 / 2} v_{\mathrm{th}} \cos \alpha_{\mathrm{lc}}$. Then, the total amount of accelerated electrons is calculated by integrating the reduced distribution function $F_{\text {acc }}\left(V_{\|}\right)$over $V_{\|}$providing

$$
\begin{aligned}
\frac{N_{\mathrm{acc}}}{N}= & \mathrm{e}^{-\left[V_{\mathrm{s}}^{2} \sin ^{2} \alpha_{\mathrm{lc}}+V_{\mathrm{e}}^{2} \tan ^{2} \alpha_{\mathrm{lc}}\right] / 2 v_{\mathrm{th}}^{2}} \\
& \times \frac{\cos \alpha_{\mathrm{lc}}}{2}\left[1+2 \Phi_{0}\left(\frac{V_{\mathrm{s}} \cos \alpha_{\mathrm{lc}}}{v_{\mathrm{th}}}\right)\right]
\end{aligned}
$$

where the function $\Phi_{0}(\xi)$ is defined by

$\Phi_{0}(\xi)=\frac{1}{\sqrt{2 \pi}} \cdot \int_{0}^{\xi} \mathrm{d} x \mathrm{e}^{-x^{2} / 2}$.

Such a beam-like distribution function (see Eq. (16)) is unstable (Krall \& Trivelpiece 1973) for exciting of Langmuir waves, which can nonlinearly convert into radio waves as mentioned in Sect. 1. The growth rate $\Gamma$ of this instability is given by

$$
\frac{\Gamma}{\omega_{\mathrm{pe}}}=\frac{1}{\sqrt{\pi}} \cdot V_{\|}^{2} \cdot \frac{\mathrm{d} F}{\mathrm{~d} V_{\|}}
$$

with $\omega_{\mathrm{pe}}=2 \pi f_{\mathrm{pe}}$ (Magelssen \& Smith 1977). Here, the full reduced distribution function is composed of that of the accelerated electrons $F_{\text {acc }}$ and the Maxwellian one

$F_{\mathrm{M}}=\frac{1}{\left(2 \pi v_{\mathrm{th}}^{2}\right)^{1 / 2}} \cdot \mathrm{e}^{-V_{\|}^{2} / 2 v_{\mathrm{th}}^{2}}$

of the background plasma in the upstream region. Inserting Eqs. (16) and (20) into Eq. (19), inspection of the resulting equation shows that the growth rate $\Gamma$ has its maximum at $V_{\text {parallel }}=V_{\mathrm{b}}-v_{\text {th }} \cdot \cos \theta$; i.e. the instability occurs at $V_{\|} \approx V_{\mathrm{b}}$ for $V_{\|}>V_{\mathrm{s}}$ and $V_{\|} \gg v_{\text {th }}$, Thus, those electrons with velocities near the beam velocity $V_{\mathrm{b}}$ are exciting Langmuir waves needed for the radio emission (see also Ball \& Melrose 2001).

\section{Discussion}

Shock-drift acceleration is very efficient for quasiperpendicular shocks, i.e. $45^{\circ}<\theta<90^{\circ}$, and produces a beam-like distribution function (see Eq. (16)) for the accelerated electrons. The beam velocity is given by $V_{\mathrm{b}}=v_{\mathrm{s}} \cdot \sec \theta \cdot\left(1+\cos ^{2} \alpha_{\mathrm{lc}}\right)$. In order to compare the theoretical results with observations, some basic parameters must be introduced. As deduced from the observations, the Alfvén-Mach number of coronal shock waves associated with type II radio bursts exceeds 1.5 (see Sect. 3). Therefore, a jump in the magnetic field and the particle number density of $B_{\text {down }} / B_{\text {up }} \approx 2$ and $N_{\text {down }} / N_{\text {up }} \approx 2$ are justified to be assumed for such shocks, respectively. Then, the loss-cone angle is calculated to be $\alpha_{\mathrm{lc}}=45^{\circ}$. The cross shock potential in the de Hoffmann-Teller frame is derived as $e \phi_{\mathrm{HT}}=1.47 k_{\mathrm{B}} T$ with $\gamma=5 / 3$ (see Eq. (10)) leading to $V_{\mathrm{e}}=7900 \mathrm{~km} \mathrm{~s}^{-1}$ for a coronal temperature of $1.4 \times 10^{6} \mathrm{~K}$. As is well-known, Langmuir waves are necessary for radio emission (Melrose 1985). In order to excite them, the electron beam velocity must 
fulfill the relationship $V_{\mathrm{b}}=v_{\mathrm{s}} \sec \theta\left(1+\cos ^{2} \alpha_{\mathrm{lc}}\right)>3^{1 / 2} v_{\text {th }}$ (Krall \& Trivelpiece 1973) requiring $\theta>81.8^{\circ}$. Consequently, the type II burst associated shock waves must have a nearly perpendicular shock geometry, if the shock accelerated electron beams are observed as "herringbones" in the solar radio radiation.

Adopting $V_{\mathrm{b}}=V_{\mathrm{HB}}=52000 \mathrm{~km} \mathrm{~s}^{-1}$ and $v_{\mathrm{s}}=V_{\mathrm{II}}=$ $760 \mathrm{~km} \mathrm{~s}^{-1}$ as typical values for the velocity of the electron beam related to the "herringbones" and the speed of the associated shock wave, respectively, the angle $\theta$ can be determined as $88.7^{\circ}$. Then, the speed $V_{\mathrm{s}}$ is $23000 \mathrm{~km} \mathrm{~s}^{-1}$. (Note that $V_{\mathrm{s}}$ is actually the shock speed in the de Hoffmann-Teller frame. It is really non-relativistic as required for the non-relativistic treatment of the shock-drift acceleration.) Finally, $2.8 \times 10^{-7}$ of all electrons in the upstream region has been accelerated according to Eq. (17).

Since $V_{\mathrm{b}} \propto v_{\mathrm{S}}$ (see Sect. 4) the velocity of the electrons related to the "herringbones" is increasing with the speed of the shock associated with the type II burst as indicated by observations (see Fig. 3). Because of

$\frac{D_{f, \mathrm{HB}}}{D_{f, \mathrm{II}}}=\frac{V_{\mathrm{b}}}{v_{\mathrm{s}}}=\sec \theta \cdot\left(1+\cos ^{2} \alpha_{\mathrm{lc}}\right)$

the angle $\theta$ can be derived as $88.9^{\circ}$ and $89.7^{\circ}$ for $D_{f, \mathrm{HB}} / D_{f, \mathrm{II}}=$ 77 (mean value) and 350 (highest value) (see Fig. 3). This result confirms the conclusion that the shock waves generating "herringbones" must have a nearly perpendiclur shock geometry, once more. Note that it is independent of the coronal density model used.

In the aforementioned estimates (see Eq. (21)) it was basically assumed that both the "herringbone" and type II burst sources are radially propagating. But, this is not true. If done correctly,

$$
\begin{aligned}
\frac{D_{f, \mathrm{HB}}}{D_{f, \mathrm{II}}} & =\frac{V_{\mathrm{b}}}{v_{\mathrm{S}}} \cdot \frac{\cos \vartheta_{\mathrm{HB}, n_{r}}}{\cos \vartheta_{\mathrm{II}, n_{r}}} \\
& =\sec \theta \cdot\left(1+\cos ^{2} \alpha_{\mathrm{lc}}\right) \cdot \frac{\cos \vartheta_{\mathrm{HB}, n_{r}}}{\cos \vartheta_{\mathrm{II}, n_{r}}}
\end{aligned}
$$

is immediately derived from Eq. (2). Here, $\vartheta_{\mathrm{HB}, n_{r}}$ and $\vartheta_{\mathrm{II}, n_{r}}$ denote the angles between the propagation direction of the "herringbone" and the type II burst source to the radial one. But, since the shock waves generating "herringbones" should be regarded as nearly perpendicular, $\vartheta_{\mathrm{HB}, n_{r}}+\vartheta_{\mathrm{II}, n_{r}} \approx 90^{\circ}$ is valid for these angles leading to the relationship

$\cos \theta=\frac{D_{f, \mathrm{II}}}{D_{f, \mathrm{HB}}} \cdot\left(1+\cos ^{2} \alpha_{\mathrm{lc}}\right) \cdot \tan \vartheta_{\mathrm{II}, n_{r}}$

from Eq. (22). Since the angle $\vartheta_{\mathrm{III}, n_{r}}$ can vary in the range $0^{\circ}-90^{\circ}, 45^{\circ}$ is chosen as a mean value for this angle, for instance. Then, $\theta=88.7^{\circ}$ is obtained by Eq. (23) for $D_{f, \mathrm{HB}} / D_{f, \mathrm{II}}=77$ (see Fig. 3). But for such an angle and $\vartheta_{\mathrm{II}, n_{r}}=$ $45^{\circ}$, a mean speed of $1100 \mathrm{~km} \mathrm{~s}^{-1}$ and $74000 \mathrm{~km} \mathrm{~s}^{-1}(=0.25 \mathrm{c})$ are derived for the shocks associated with the type II bursts and the electrons related to the "herringbones" from the observations (see Sect. 2), respectively.

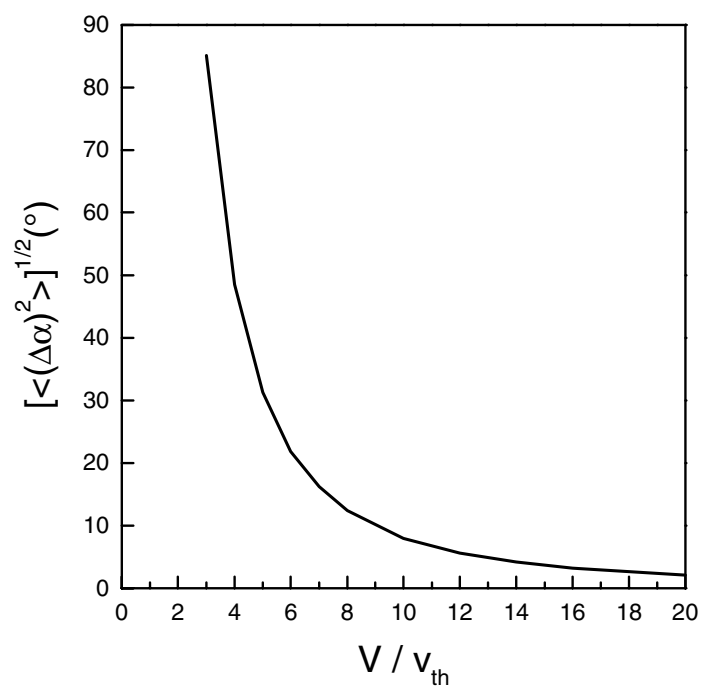

Fig. 5. Dependence of the mean deflection angle $\left[\left\langle(\Delta \alpha)^{2}\right\rangle\right]^{1 / 2}$ (see Eq. (24)) on the particle velocity $V$ normalized to the thermal electron speed $v_{\text {th }}$.

In this presented approach it was basically assumed that a shifted loss-cone distribution is established for the accelerated electrons due to shock-drift acceleration in the upstream region of the shock wave. This requires the electrons to behave as collisionless particles. In order to check that, the mean angle of deflection due to Coulomb collisions after a propagation path $\mathrm{L}$ is investigated in a fully ionized plasma with the electron number density $N$ and temperature $T$. This angle is determined by

$\left\langle(\Delta \alpha)^{2}\right\rangle=\frac{8 \pi N L e^{4}}{m_{\mathrm{e}}^{2} V^{4}} \cdot \ln \left[\sqrt{\frac{k_{\mathrm{B}} T}{4 \pi e^{2} N}} \cdot \frac{m_{\mathrm{e}} V^{2}}{e^{2}}\right]$

(Krall \& Trivelpiece 1973; Estel \& Mann 1999). Here, $V$ denotes the full electron velocity, i.e. $V=\left(V_{\|}^{2}+V_{\perp}^{2}\right)^{1 / 2}$. Since the "herringbones" have an extension of about $10 \mathrm{MHz}$ in the dynamic radio spectrum (see Sect. 2), the electrons related to the "herringbones" have a mean travel distance of $60000 \mathrm{~km}$ from the associated shock waves. This corresponds to the radial distance between the $54 \mathrm{MHz}$ and $44 \mathrm{MHz}$ levels in the corona according to Eq. (3). Figure 5 represents the behaviour of the mean deflection angle $\left[\left\langle(\Delta \alpha)^{2}\right\rangle\right]^{1 / 2}$ versus the particle velocity $V / v_{\text {th }}$ according to Eq. (24). Here, $N=3.62 \times 10^{7} \mathrm{~cm}^{-3}$, $T=1.4 \times 10^{6} \mathrm{~K}$, and $L=60000 \mathrm{~km}$ have been used for the calculations. Thus, particles with a velocity $V>5 v_{\text {th }}$ can be regarded as collisionless in the case under consideration, since the mean deflection angle is smaller than $30^{\circ}$ for them. That is really the case for the electrons related to the "herringbones", since their typical (radial) velocity of about $52000 \mathrm{~km} \mathrm{~s}^{-1}$ is greater than $5 v_{\mathrm{th}}=23000 \mathrm{~km} \mathrm{~s}^{-1}$.

\section{Summary}

"Herringbones" appear as special fine structures within solar type II radio bursts, which are regarded as radio signatures of shock waves travelling through the corona. They are considered 
as electron beams generated by the shock wave associated with the type II burst. Assuming that shock-drift acceleration is responsible for generation of "herringbones", the associated shock must be nearly perpendicular. The electrons accelerated by such a mechanism form a shifted loss-cone distribution in the upstream region of the shock. Such a distribution provides a pure beam-like distribution for the reduced distribution function. Since the beam velocity is proportional to $\sec \theta$ (see Eq. (16)), a decrease in the angle $\theta$ below $85^{\circ}$ would dramatically decrease the beam velocity to values at which Coulomb collisions become dominant. This property could be the origin of the bursty occurrence of the "herringbones" in dynamic radio spectra during type II bursts, since the angle $\theta$ is expected to be locally fluctuating at real shocks like type II burst sources. The energies of the electron beams related to the "herringbones" cover a broad range $0.2-80 \mathrm{keV}$, where their energies have typical values of about $7 \mathrm{keV}$. About $10^{-7}$ (see Eq. (17)) of all electrons in the upstream region have been accelerated.

With respect to the type III radio bursts, the energetic electrons are considered to be initially produced as a population governed by a power law. Beam-like distributions are established due to the spatio-temporal evolution of such an electron population; i.e. the faster electrons are running away from the slower ones (see e.g. Magelssen \& Smith 1977 and Grognard 1985). Thus, different parts of these electrons are responsible for the type III radio burst radiation at different places during their propagation, i.e. at different frequencies. Signatures of such a process were really observed during the event on December 27, 1994 (Mann et al. 1999b). In contrast to that, a pure beam-like distribution (see Eq. (16)) of the "herringbone"related electrons is intially formed by shock drift acceleration. This difference in the process generating type III bursts and "herringbones" could be the origin of the morphological differences between both phenomena as reported by Cairns \& Robinson (1987) and Zlobec et al. (1993). In dynamic radio spectra type III bursts typically show a "fan"-shaped structure; i.e., the duration at a fixed frequency increases with decreasing frequency. In this sample of "herringbones" the duration stays constant over the whole extension of the individual "herringbones" (see frame B and C in Fig. 1). That result contrasts to the observations by Cairns \& Robinson (1987), who reported on a "thorn"-shaped structure of the "herringbones". But, the most important difference between the two is that the whole frequency range of the "herringbones" is very small, i.e. $\Delta f_{\mathrm{HB}} \approx 10 \mathrm{MHz}$ (see Fig. 4), in contrast to the type III bursts, which can cover a much broader frequency range.

Acknowledgements. This work was supported by the German Deutsche Forschungsgemeinschaft (DFG) under project number MA 1376/14-1.

\section{References}

Aurass, H. 1996, in Coronal Physics from Radio and Space Observations, ed. G. Trottet, Lecture Notes in Physics (Heidelberg: Springer Verlag), 135

Aurass, H., Hofmann, A., \& Urbarz, H.-W. 1998, A\&A, 334, 289
Bale, S. D., Reiner, M. J., Bougeret, J. L., et al. 1999, Geophys. Res. Lett., 26, 1573

Ball, L., \& Melrose, D. B. 2001, Publ. Astron. Soc. Austr., 18, 361

Benz, A. O. 1987, Sol. Phys., 111, 1

Cairns, I. H., \& Robinson, R. D. 1987, Sol. Phys., 111, 365

Cairns, I. H., \& Robinson, P. A. 1997, Geophys. Res. Lett., 24, 369

Cairns, I. H., \& Robinson, P. A. 1999, Phys. Rev. Lett., 82, 3066

Cairns, I. H., \& Robinson, P. A. 2000, Geophys. Rev. Lett., 27, 61

Cairns, I. H., Knock, S. A., Robinson, P. A., \& Kunic, Z. 2003, Space Sci. Rev., 107, 27

Dulk, G. A., \& McLean, D. J. 1978, A\&A, 66, 315

Estel, C., \& Mann, G. 1999, A\&A, 345, 276

Goodrich, C. C., \& Scudder 1984, J. Geophys. Res., 89, 6654

Gopalswamy, N., Kaiser, M. L., Sato, J., \& Pick, M. 2000, in High Energy Solar Physiscs, ed. R. Ramaty \& N. Mandzhavidze, PASP Conf. Ser., 206, 351

Grognard, R. J.-M. 1985, in Solar Radio Physics, ed. D. J. McLean \& N. R. Labrum (Cambridge: Cambridge Univ. Press), 253

Holman, G. D. 1985, ApJ, 293, 584

Holman, G. D., \& Pesses, M. E. 1983, ApJ, 267, 837

Kahler, S. W. 1994, ApJ, 428, 837

Kennel, C. F., Edmiston, J. P., \& Hada, T. 1985, in Collisionless Shocks in the Heliosphere: A Tutorial Review, ed. R. G. Stone \& B. T. Tsurutani, Geophysical Monograph, 34 (Washington D.C.; AGU), 1

Kirk, J. G. 1994, in Plasma Astrophysics, ed. A. O. Benz \& T., J.-L. Courvoisier (Springer Verlag), 225

Klassen, A., Aurass, H., Klein, K.-L., Hofmann, A., \& Mann, G. 1999, A\&A, 343, 287

Klassen, A., Aurass, H., Mann, G., \& Thompson, B. J. 2000, A\&AS, 141,357

Klein, K.-L., \& Trottet, G. 2001, Space Sci. Rev., 95, 215

Klein, K.-L., Khan, J. I., Vilmer, N., Delouis, J.-M., \& Aurass, H. 1999, A\&A, 346, L53

Knock, S. A., Cairns, I. H., Robinson, P. A., \& Kunic, Z. 2001, J. Geophys. Res., 106, 25041

Knock, S. A., Cairns, I. H., Robinson, P. A., \& Kunic, Z. 2003, J. Geophys. Res., 108, 1126

Koutchmy, S. 1994, Adv. Space Res., 14(4), 29

Krall, N. A., \& Trivelpiece, A. W. 1973, Principles of Plasma Physics (New York: McGraw-Hill), 621

Kunic, Z. Cairns, I. H., \& Knock, S. A. 2001, J. Geophys. Res., 107, 101029

Leroy, M. M., \& Mangeney, A. 1984, Ann. Geophys., 2, 449

Lin, R. P. 1974, Space Sci. Rev., 16, 412

Litvinenko, Y. E. 2000, in High Energy Solar Physics, ed. R. Ramaty \& N. Mandzhavidze, ASPC Conf. Ser., 206, 167

Magelssen, G. R., \& Smith, D. F. 1977, Sol. Phys., 55, 211

Mann, G. 1995, in Coronal Magnetic Energy Release, ed. A. O. Benz \& A. Krüger, Lecture Notes in Physics (Heidelberg: Springer Verlag), 183

Mann, G., \& Classen, H. T. 1995, A\&A, 295, 775

Mann, G., \& Klassen, A. 2002, in Proc. 10th European Solar Phys. Meeting, ESA SP-506, 245

Mann, G., Aurass, H., Voigt, W., \& Paschke, J. 1992, in Proc. 1st SOHO Workshop, ESA-SP, 348, 129

Mann, G., Jansen, F., MacDowall, R. J., Kaiser, M. L., \& Stone, R. G. 1999, A\&A, 348, 614

Mann, G., Classen, H. T., \& Motschmann, U. 2001, J. Geophys. Res., 106(25), 323

Mann, G., Klassen, A., Aurass, H., \& Classen, H. T. 2003, A\&A, 400, 329

Melrose, D. 1985, in Solar Radio Physics, ed. D. J. McLean \& N. R. Labrum (Cambridge: Cambridge Univ. Press), 177 
Melrose, D. 1994, in Plasma Astrophysics, ed. A. O. Benz \& T. J.-L. Courvoisier (Springer Verlag), 123

Miller, J. A. 1997, ApJ, 491, 939

Nelson, G. S., \& Melrose, D. 1985, in Solar Radio Physics, ed. D. J. McLean \& N. R. Labrum (Cambridge: Cambridge Univ. Press), 333

Newkirk, G. A. 1961, ApJ, 133, 983

Reames, D. V., Barbier, L. M., \& Ng, C. K. 1996, ApJ, 466, 473

Roberts, J. A. 1959, Astralien J. Phys., 12, 322

Schlickeiser, R. 1984, A\&A, 136, 227

Stewart, R. T., Howard, R. A., Hansen, F., Gergely, T., \& Kundu, M. R. 1974a, Sol. Phys., 36, 219
Stewart, R. T., McCabe, M., Koomen, M. J., Hansen, F., \& Dulk, G. 1974b, Sol. Phys., 26, 203

Suzuki, S., \& Dulk, G. A. 1985, in Solar Radio Physics, ed. D. J. McLean \& N. R. Labrum (Cambridge: Cambridge Univ. Press), 289

Uchida, Y. 1960, PASJ, 12, 376

Uchida, Y., Altschuler, D., \& Newkirk, G. 1973, Sol. Phys., 28, 495

Vršnak, B., Ruždjak, V., \& Aurass, H. 1995 Sol. Phys., 158, 331

Wild, J. P., \& McCready, L. L. 1950, Austr. J. Sci. Res. Ser. A, 3, 387

Wu, C. S. 1984, J. Geophys. Res., 89, 8857

Zlobec, P. Messerotti, M., Karlicky, M. \& Urbarz, H. 1993, Sol. Phys., 144,373 